\title{
Targeting glioma-initiating cells via the tyrosine metabolic pathway
}

\author{
*Daisuke Yamashita, MD, PhD, ${ }^{1}$ Joshua D. Bernstock, MD, PhD, ${ }^{2}$ Galal Elsayed, MD, ${ }^{1}$ \\ Hirokazu Sadahiro, MD, PhD, ${ }^{1,3}$ Ahmed Mohyeldin, MD, ${ }^{4}$ Gustavo Chagoya, MD, ${ }^{1}$ Adeel llyas, MD, ${ }^{1}$ \\ James Mooney, MD, ${ }^{1}$ Dagoberto Estevez-Ordonez, MD, ${ }^{1}$ Shinobu Yamaguchi, ${ }^{1}$ Victoria L. Flanary, ${ }^{1}$ \\ James R. Hackney, MD, ${ }^{5}$ Krishna P. Bhat, PhD, ${ }^{6}$ Harley I. Kornblum, MD, PhD, ${ }^{7,8,13}$ \\ Nicola Zamboni, PhD, ${ }^{9}$ Sung-Hak Kim, PhD, ${ }^{1,10,11}$ E. Antonio Chiocca, MD, PhD, ${ }^{2}$ and \\ Ichiro Nakano, MD, PhD'1,12
}

Departments of ${ }^{1}$ Neurosurgery and ${ }^{5} \mathrm{Pathology} ;{ }^{12} \mathrm{O}$ 'Neal Comprehensive Cancer Center, University of Alabama at Birmingham, Alabama; ' $D$ epartment of Neurosurgery, Brigham and Women's Hospital, Harvard Medical School, Boston, Massachusetts; ${ }^{3}$ Department of Neurosurgery, Yamaguchi University School of Medicine, Ube, Yamaguchi, Japan; ${ }^{4}$ Department of Neurological Surgery, The Ohio State University, Wexner Medical Center, Columbus, Ohio; ${ }^{6}$ Department of Translational Molecular Pathology and Brain Tumor Center, The University of Texas MD Anderson Cancer Center, Houston, Texas; ${ }^{7}$ Departments of Psychiatry and Biobehavioral Sciences, Semel Institute for Neuroscience and Human Behavior; ${ }^{8}$ Intellectual and Developmental Disabilities Research Center, Semel Institute for Neuroscience and Human Behavior; ${ }^{33 B}$ Broad Stem Cell Research Center, David Geffen School of Medicine at UCLA, Los Angeles, California; Institute of Molecular Systems Biology, ETH Zurich, Zurich, Switzerland; ${ }^{10}$ Department of Animal Science, College of Agriculture and Life Sciences, Chonnam National University, Gwangju; and ${ }^{11}$ Gwangju Center, Korea Basic Science Institute, Gwangju, Republic of Korea

OBJECTIVE Despite an aggressive multimodal therapeutic regimen, glioblastoma (GBM) continues to portend a grave prognosis, which is driven in part by tumor heterogeneity at both the molecular and cellular levels. Accordingly, herein the authors sought to identify metabolic differences between GBM tumor core cells and edge cells and, in so doing, elucidate novel actionable therapeutic targets centered on tumor metabolism.

METHODS Comprehensive metabolic analyses were performed on 20 high-grade glioma (HGG) tissues and 30 gliomainitiating cell (GIC) sphere culture models. The results of the metabolic analyses were combined with the Ivy GBM data set. Differences in tumor metabolism between GBM tumor tissue derived from within the contrast-enhancing region (i.e., tumor core) and that from the peritumoral brain lesions (i.e., tumor edge) were sought and explored. Such changes were ultimately confirmed at the protein level via immunohistochemistry.

RESULTS Metabolic heterogeneity in both HGG tumor tissues and GBM sphere culture models was identified, and analyses suggested that tyrosine metabolism may serve as a possible therapeutic target in GBM, particularly in the tumor core. Furthermore, activation of the enzyme tyrosine aminotransferase (TAT) within the tyrosine metabolic pathway influenced the noted therapeutic resistance of the GBM core.

CONCLUSIONS Selective inhibition of the tyrosine metabolism pathway may prove highly beneficial as an adjuvant to multimodal GBM therapies.

https://thejns.org/doi/abs/10.3171/2019.11.JNS192028

KEYWORDS high-grade glioma; glioblastoma; metabolism; heterogeneity; nitrogen metabolism; tyrosine aminotransferase; oncology

$\mathrm{D}$ ESPITE multimodal therapies, glioblastoma (GBM) continues to portend an extremely poor clinical prognosis with an estimated median survival of less than 2 years. ${ }^{38}$ Tumor genetic and transcriptional heterogeneity is a critical contributor to GBM therapeutic resilience. ${ }^{22}$ Thus, it has become apparent that both histopathological and transcriptional analyses of tumor heterogeneity will be fundamental to understanding GBM and developing novel therapeutic targets. ${ }^{20,30}$ In line with such thinking, metabolic analysis of GBM has also emerged as

ABBREVIATIONS CL = classical; GBM = glioblastoma; $\mathrm{GIC}$ = glioma-initiating cell; $\mathrm{HGG}$ = high-grade glioma; IDH = isocitrate dehydrogenase; IHC = immunohistochemistry; Ivy GAP = Ivy Glioblastoma Atlas Project; MES = mesenchymal; PCA = principal component analysis; PN = proneural.

SUBMITTED July 26, 2019. ACCEPTED November 19, 2019.

INCLUDE WHEN CITING Published online February 14, 2020; DOI: 10.3171/2019.11.JNS192028.

* D.Y. and J.D.B. contributed equally to this work. 
a promising strategy in the elucidation of actionable molecular targets..$^{3,36}$

Metabolic analysis is centered on the quantitative assessment of endogenous metabolites within a biological system. ${ }^{13}$ It is known that cancer cells modify genetic and epigenetic mechanisms related to their metabolism in an effort to adapt to their harsh microenvironments. ${ }^{9,21,39}$ Historically, the discovery of increased glycolysis within cancer cells, better known as the "Warburg effect," established metabolomics. ${ }^{42}$ Since this monumental discovery, numerous studies have revealed a myriad of metabolic alterations within cancer, such as dysregulated uptake of glucose and amino acids, increased demand for nitrogen, and alterations in metabolite-driven gene regulation..$^{427,32}$ In glioma, key proteins associated with metabolic changes include the enzyme isocitrate dehydrogenase (IDH) and the transcription factor hypoxia-inducible factor 1 alpha $(\mathrm{HIF}-1 \alpha){ }^{44,45}$ Of note, other metabolites and metabolic pathways have been implicated in GBM progression and/or therapeutic resistance. ${ }^{1,23,25}$ Taking these factors into consideration, it has become abundantly clear that to understand high-grade glioma (HGG) pathobiology, cancer metabolism and its relationship to inter- and/or intratumoral heterogeneity must be further explored. ${ }^{28}$ Therefore, we hypothesized that activation of metabolic pathways would accelerate inter-/intratumoral heterogeneity as well as therapeutic resistance.

Herein, we performed comprehensive metabolic analyses on 20 HGG tissues and 30 glioma-initiating cell (GIC) sphere culture models and then focused on activated metabolic pathways. The results of our metabolic analyses were combined with the Ivy GBM data set. ${ }^{33}$ Differences in metabolism between the GBM tumor tissue derived from within the contrast-enhancing region (i.e., tumor core) and that within the peritumoral normal/reactive brain lesions (i.e., tumor edge) were sought and explored. ${ }^{28,30,31}$ Such changes were ultimately confirmed at the protein level via immunohistochemistry (IHC).

\section{Methods}

\section{Ethics}

All work related to human tissue was performed at the University of Alabama at Birmingham under an institutional review board-approved protocol compliant with guidelines set forth by the National Institutes of Health. Patient-derived specimens were provided to the corresponding scientists after de-identification of the original tumors.

\section{GBM-Derived (Neuro)Sphere Cultures}

The sphere lines starting with LA (Los Angeles) were established by the laboratory led by Harley Kornblum (UCLA). The lines starting with GSC (glioma stem cell) or R (University of Regensburg) were established or shared by the laboratory led by Krishna Bhat (MD Anderson Cancer Center). OS157 was derived from the Nakano laboratory. Glioma sphere cultures from clinical samples were cultured in DMEM/F12 medium containing 2\% B27 supplement (\% vol), $2.5 \mathrm{mg} / \mathrm{ml}$ heparin, $20 \mathrm{ng} / \mathrm{ml}$ basic fibroblast growth factor (bFGF), and $20 \mathrm{ng} / \mathrm{ml}$ epidermal growth factor (EGF). The bFGF and EGF reagents were added twice a week, and the culture medium was replaced every 10 days. Experiments with (neuro)spheres were performed with lines that were cultured for $<40$ passages since their initial establishment. Short tandem repeat analysis was performed to identify human cell lines derived from the tissue of a single individual.

\section{Metabolomics}

Approximately $1 \mathrm{mg}$ of human GBM tissue or $10^{6}$ cells were collected for analysis. Resected tissue was classified as having originated from the tumor core (i.e., within the contrast-enhancing rim) or from the tumor edge (i.e., peritumoral FLAIR signal). The sample was pulverized with a pestle and immediately placed in ice-cold methanol. After homogenization, $4 \mathrm{ml}$ of ice-cold methanol was added, and the sample was centrifuged at $10,000 \mathrm{rpm}$ at $4^{\circ} \mathrm{C}$ for 10 minutes. Extracted metabolites were analyzed by flow injection-time-of-flight mass spectrometry on an Agilent 6550 Q-TOF instrument operated in the negative mode, as previously described. ${ }^{11}$ Detectable ions were putatively annotated by matching measured mass-to-charge ratios with theoretical masses of compounds listed in the Human Metabolome Database v3.0 using a tolerance of $0.001 \mathrm{amu}^{43}$ Pathway definition of differentially abundant metabolites was performed using the Small Molecule Pathway Database. ${ }^{10} \mathrm{p}$ values were calculated using a two-tailed, heteroscedastic t-test and were adjusted for the false discovery rate (FDR) according to the Benjamini-Hochberg procedure. All calculations were done in MATLAB.

\section{Ivy Glioblastoma Atlas Project RNA Sequencing Analysis}

For RNA sequencing analysis, raw gene-level values of fragments per kilobase of transcript per million mapped reads (FPKM) and sample information were downloaded from the Ivy Glioblastoma Atlas Project (Ivy GAP) website (http://glioblastoma.alleninstitute.org). The database includes gene expression data for multiple structural features commonly identified in GBM enriched by laser capture microdissection.

\section{Immunohistochemistry}

IHC was performed as has been previously described. ${ }^{28}$ Briefly, tumors embedded in paraffin blocks were deparaffinized, and hydrated via progression through an ethanol series. After microwave-mediated antigen retrieval in an IHC antigen retrieval solution, $\mathrm{pH} 6$ (Invitrogen), slides were incubated in a $0.3 \%$ hydrogen peroxide solution for 15 minutes at room temperature to inhibit intrinsic peroxidase activity. Next, samples were blocked with a serumfree protein blocking solution (Thermo Fisher Scientific) and incubated with the corresponding primary antibodies overnight at $4^{\circ} \mathrm{C}$. Slides were then stained with SignalStain Boost IHC Detection Reagent (Cell Signaling Technology) and visualized with 3,3'-diaminobenzidine (DAB) peroxidase substrate kit (Vector Laboratories Inc.). Images were captured using an EVOS FL microscope (Advanced Microscopy Group).

\section{Statistical Analysis}

Statistical analyses were performed using XLSTAT 2018.5 (Addinsoft), SPSS statistical package version 25 


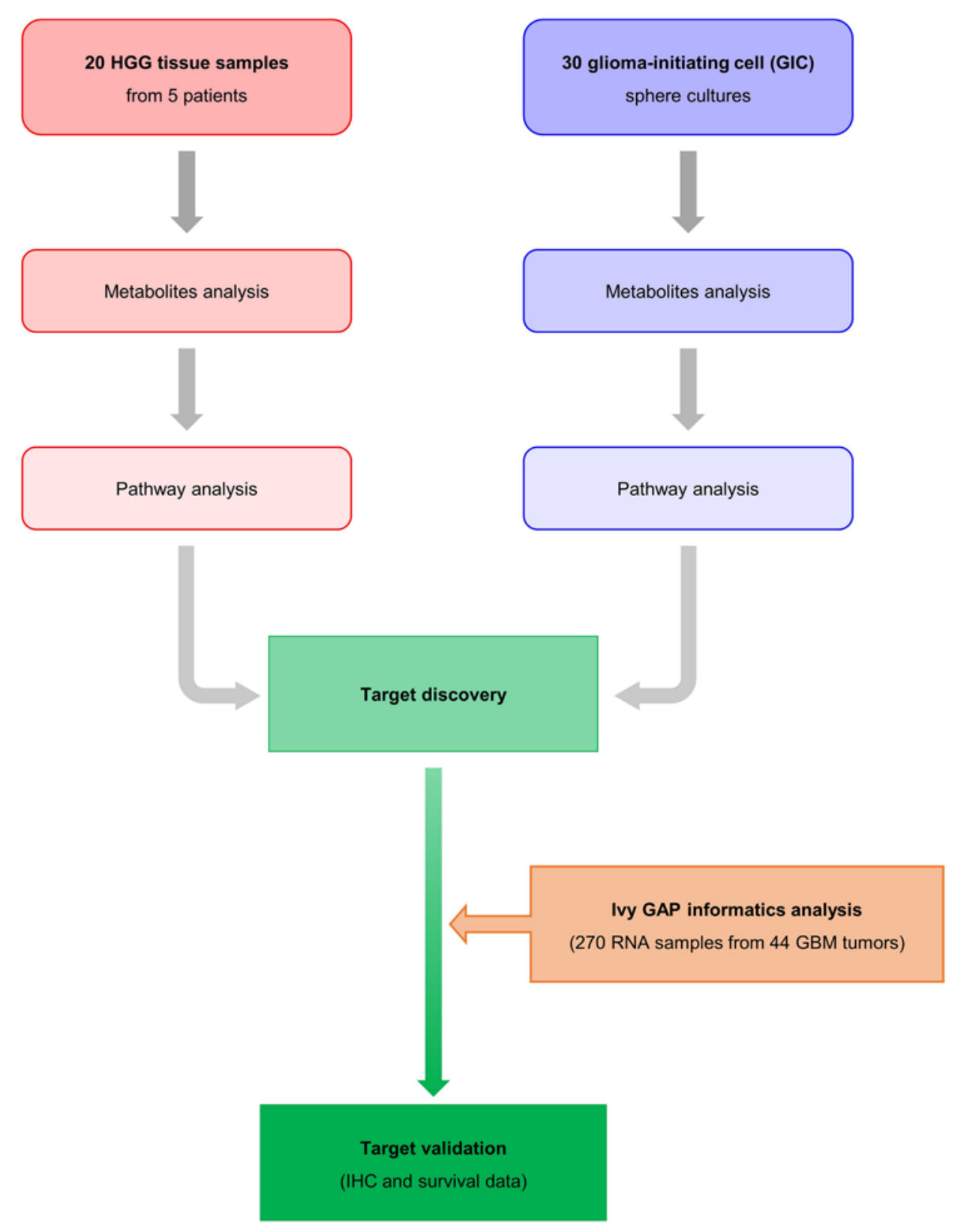

FIG. 1. Schematic outlining procedural identification of viable targets and validating assay for evaluation of clinical relevance.

(IBM Corp.), and Prism 7.0 software (GraphPad Software Inc.). All data were presented as the mean \pm standard deviation. The $\mathrm{p}$ values $<0.05$ were considered to be statistically significant.

\section{Results}

\section{Characterization of Intra- and Intertumoral Metabolic Heterogeneity in HGGs}

In order to establish a data analysis pipeline for HGG analyses, we designed a stepwise procedural approach to identify metabolic pathways that are selectively activated in the HGG cells (Fig. 1). First, the senior author resected 20 HGG tissues from 5 patients (4 with $I D H$-wt GBM and 1 with IDH-mutant grade III anaplastic oligodendroglioma) and provided tissues for the metabolic analysis (Fig. 2A). Representative preoperative Gd-enhancing T1weighted images demonstrated a heterogeneously enhancing solitary lesion with areas of hypointensity, ventricular compression, and midline shift (Fig. 2B). We fractionalized each tumor tissue sample into 4 or 5 pieces (Fig. 2C). Histopathological evaluation confirmed that these tumor samples contained features typical of GBM, including pseudopalisading with necrosis, microvascular proliferation, and hypercellular proliferation (Fig. 2D). Mass spectroscopy was ultimately employed to identify metabolites within the resected tissues. K-means clustering of the identified metabolite profiles clearly separated these tis- 
A

\begin{tabular}{cccc}
\multicolumn{4}{c}{ Characterization of tumor tissue samples } \\
\hline $\begin{array}{c}\text { Patient } \\
\text { ID }\end{array}$ & Pathological diagnosis & $\begin{array}{c}\text { IDH } \\
\text { status }\end{array}$ & $\begin{array}{c}\text { Number of } \\
\text { pieces }\end{array}$ \\
\hline P1 & Anaplastic oligodendroglioma (Grade III) & Mut & 4 \\
P2 & Glioblastoma (Grade IV) & WT & 5 \\
P4 & Glioblastoma (Grade IV) & WT & 4 \\
P5 & Glioblastoma (Grade IV) & WT & 4 \\
P6 & Glioblastoma (Grade IV) & WT & 3 \\
\hline
\end{tabular}

C

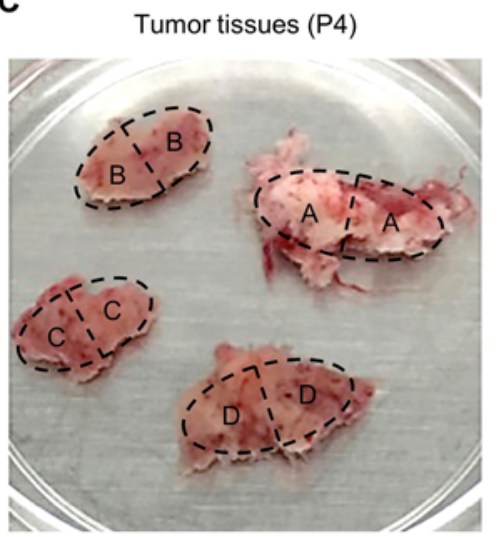

D
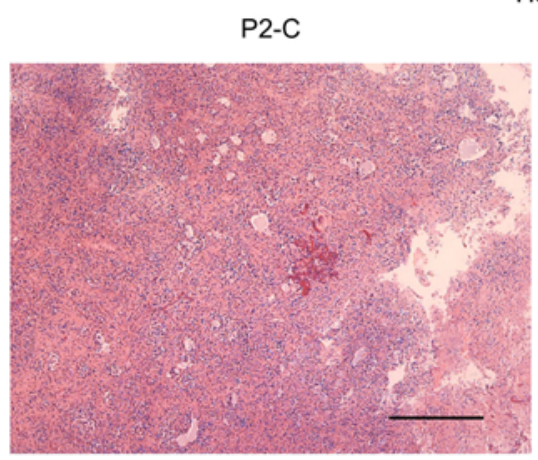

$\mathrm{H} \& \mathrm{E}$

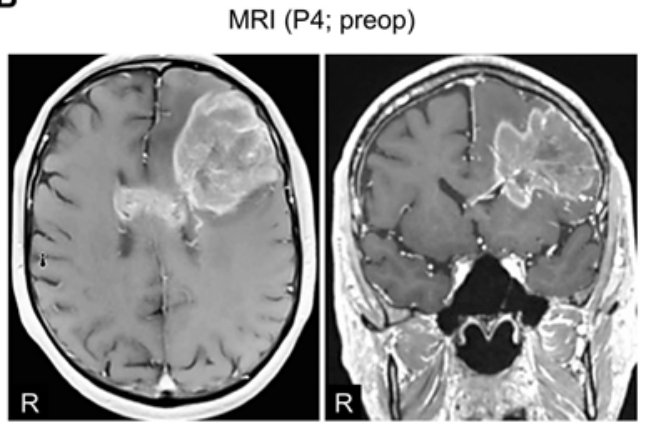

F

E

Consensus clustering
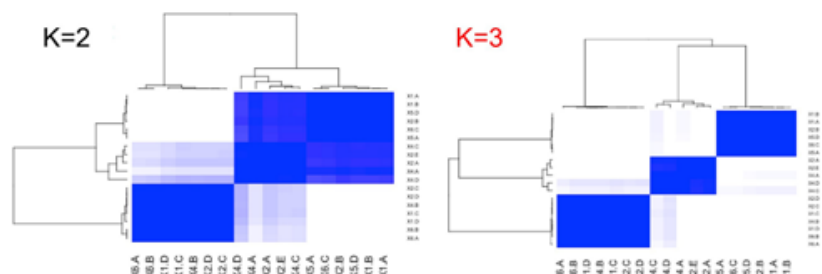

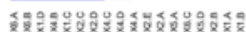
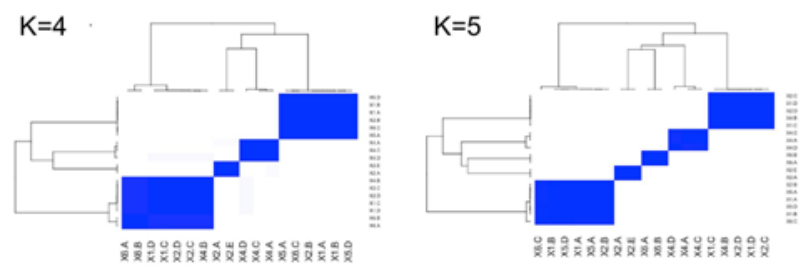

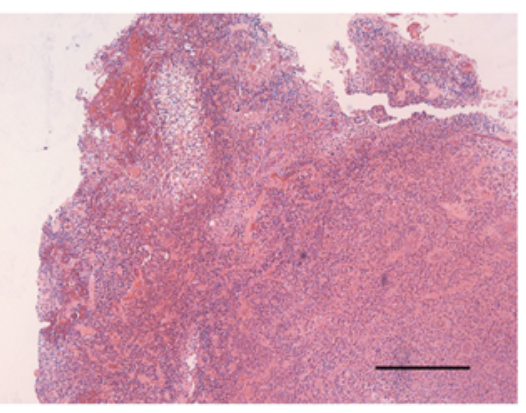

G

Distribution of three groups in each patient
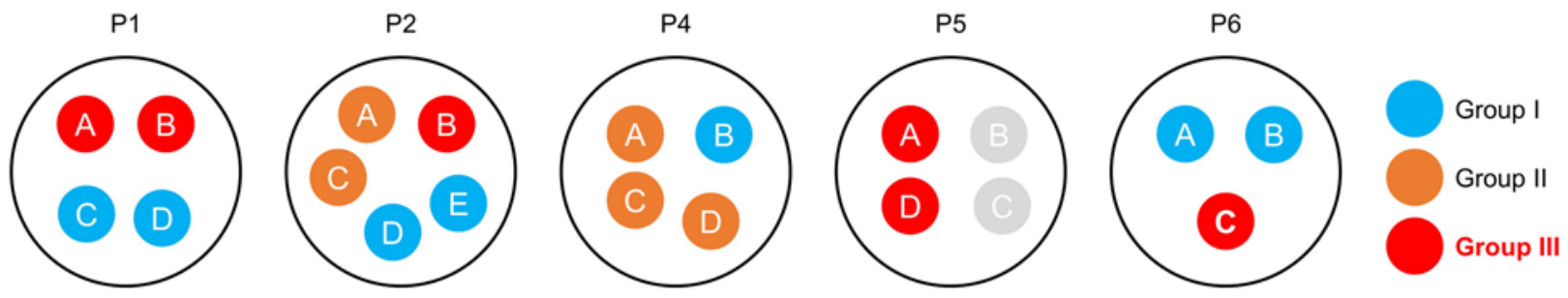

FIG. 2. Metabolic characterization of surgically derived tumor tissues from patients with HGG. A: Table detailing the relevant tumor tissue sample data for 1 anaplastic oligodendroglioma and 4 GBM patients. B: Preoperative T1-weighted MR images obtained in patient 4 (P4), depicting a left frontal solitary lesion with heterogeneous, Gd-positive enhancement in a postcontrast study. FIG. 2. (continued) $\rightarrow$ 
FIG. 2. C: Postresection gross tumor from P4 partitioned into 4 distinct samples. D: Representative photomicrographs with $H$ \& $E$ staining of tumor samples from P2 (left) and P6 (right) demonstrating typical GBM features. Bar $=200 \mu \mathrm{m}$. E: Consensus clustering of the identified metabolite profiles from tumor samples. $\mathrm{K}=$ number of clusters. F: Principal component analysis (PCA) of all tumor samples according to spatially distinctive profiles. PC1 = first principal component; PC2 = second principal component. G: Distribution of 3 groups in all tumor samples.

sues into 3 subgroups with at least 3 samples in each group (Fig. 2E). Principal component analysis (PCA) of metabolite data according to consensus clustering identified distinct metabolite signatures (Fig. 2F). Of note, all 5 tumors, including the WHO grade III tumor, were composed of multiple subgroups indicative of intra- and intertumoral metabolic heterogeneity (Fig. 2G). Interestingly, among the subgroups, we found that group III had the largest distinct and activated metabolic expression pattern in comparison to the other 2 groups (Fig. 3A). Subsequent pathway analysis identified 6 significantly activated pathways in group III; the nitrogen metabolism pathway was shown to be the most activated (Fig. 3B and C).

\section{Characterization of Intracellular Metabolic Heterogeneity in GICs}

Next, we used our 30 well-characterized GIC models derived from 30 GBM patients (Fig. 4A). Transcriptional subtyping of these GICs with cDNA microarray confirmed that these models are composed of all 3 subtypes of GBM: proneural (PN), mesenchymal (MES), and classical (CL). K-means clustering of the mass spectroscopy-based metabolic profiling distinctively identified 2 subtypes within these GICs with at least 3 samples in each group (Fig. 4B). As was the case in HGG tissue, PCA of metabolite data according to consensus clustering again identified distinct metabolite signatures (Fig. 4C). With regard to the 3 subtypes of GBM, group I included more PN and fewer MES subtypes as compared to those in group II; the rate of CL subtype was almost the same in both groups (Fig. 4D). Group II showed substantially higher metabolic molecular expression than group I, suggesting that the MES subtype has elevated metabolic activity as compared to the CL and PN subtypes (Fig. 5A). Pathway analysis identified 9

significantly activated pathways within group II, among

B

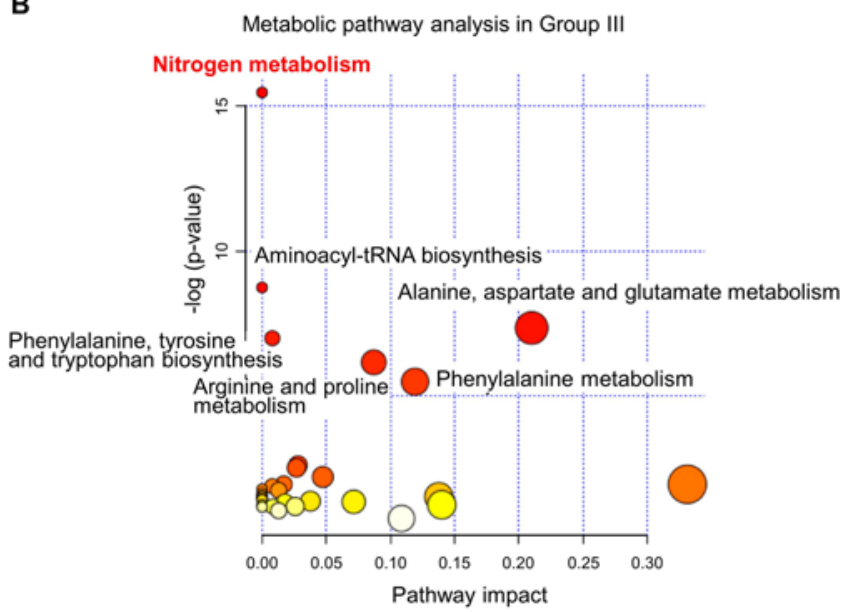

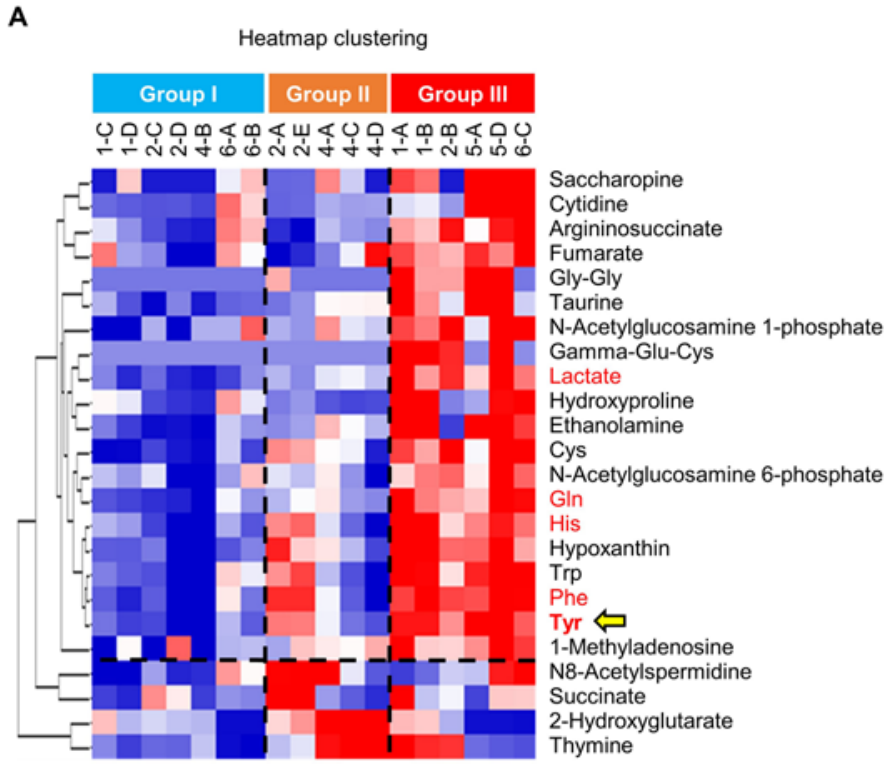

Saccharopin

Argininosuccinate

Fumarate

Gly-Gly

$\mathrm{N}$-Acetylglucosamine 1-phosphat

Gamma-Glu-Cys

Lactate

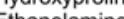

Ethan

$\mathrm{N}$-Acetylglucosamine 6-phosphate 
A

\begin{tabular}{ccc}
\multicolumn{3}{c}{ Subtypes of 30 GIC spheres } \\
\hline $\begin{array}{c}\text { Proneural } \\
(\mathrm{PN})\end{array}$ & $\begin{array}{c}\text { Mesenchymal } \\
(\mathrm{MES})\end{array}$ & $\begin{array}{c}\text { Classical } \\
(\mathrm{CL})\end{array}$ \\
\hline GSC11 & LA401 & LA411 \\
R28 & R8 & LA254 \\
R54 & LA336 & LA390 \\
LA157 & LA250 & LA227 \\
LA408 & LA350 & LA244 \\
LA348 & LA248 & LA417 \\
R18 & LA339 & LA374 \\
LA217 & LA345 & \\
GSC23 & LA412 & \\
GSC7-11 & GSC20 & \\
OS157 & GSC28 & \\
& GSC267 & \\
\hline
\end{tabular}

B
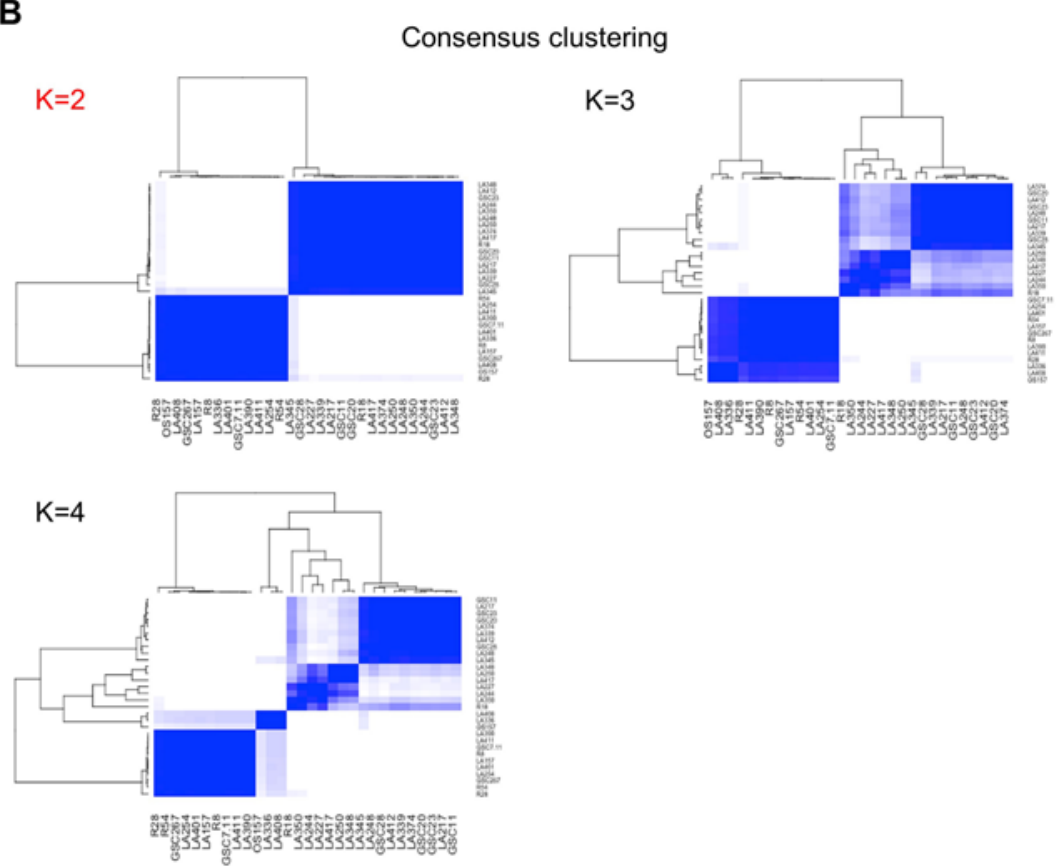

C

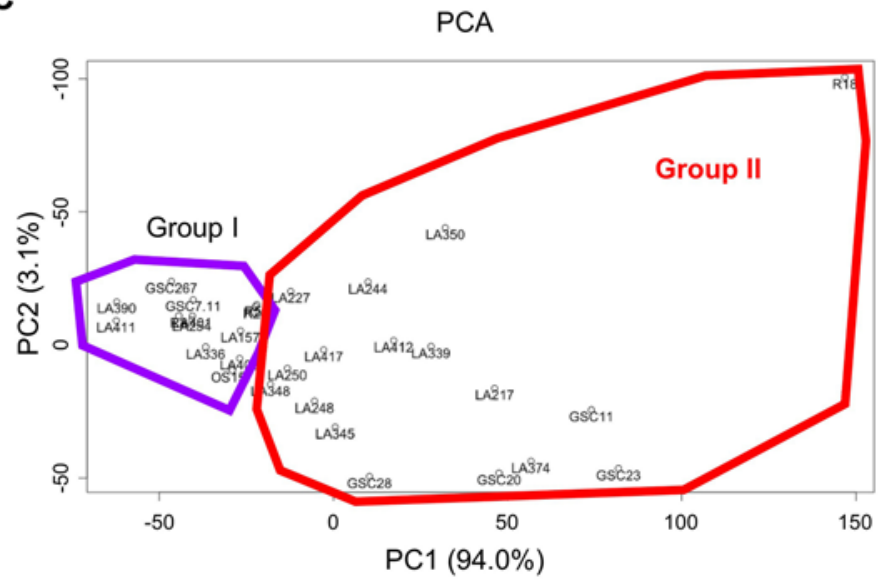

D
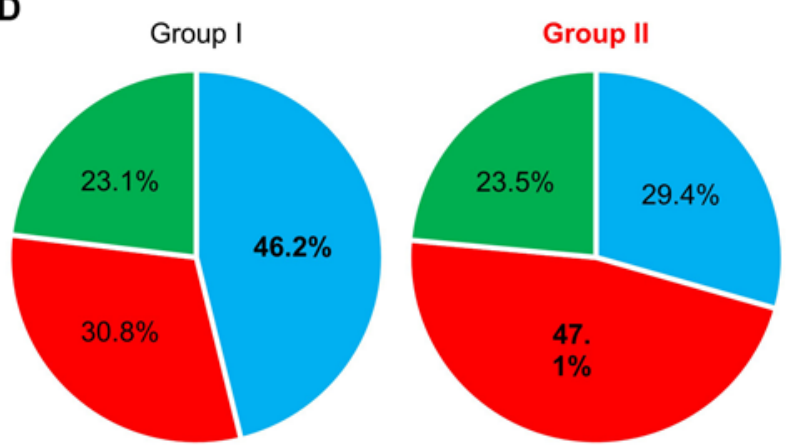

PN

MES

CL

FIG. 4. Metabolic characterization of GIC spheres. A: Table detailing relevant data for $30 \mathrm{GIC}$ spheres separated into 3 GBM subtypes: proneural (PN), mesenchymal (MES), and classical (CL). B: Consensus clustering of the identified metabolite profiles from GIC spheres. C: PCA of all GIC spheres according to spatially distinctive profiles. D: Distribution of 3 GBM subtypes in both group I and group II GIC spheres.

which nitrogen metabolism proved to be the second most activated (Fig. 5B and C).

\section{Nitrogen Pathway Activated in Both Hypermetabolic HGGs and GICs}

In comparing the results derived from HGG tissue (Fig. 3) and GIC models (Fig. 5), we found that both group III (tumor) and group II (GIC) displayed a high level of 5 metabolites, which included tyrosine (Fig. 6A). Similarly, we also identified 5 activated pathways, and the nitrogen metabolism pathway was included in both groups (Fig. 6B). Although all 5 metabolites had significant increases in both HGG tissue and GICs, tyrosine had the most significant relative increase in both comparisons: group I ver- sus II, group I versus III, and group II versus III of HGG tissues (Fig. 6C and D). In addition, the map of nitrogen metabolism showed that 4 of these metabolites had roles within this pathway (Fig. 6E). Therefore, our remaining work focused on nitrogen and tyrosine metabolism.

\section{Spatial Metabolic Heterogeneity of Tyrosine Metabolism}

One major challenge related to the metabolic data is the lack of spatial information with regard to the metabolic profiles that are derived from tumor tissues and GICs. To overcome this challenge, the senior author performed navigation-based biopsies of tumor core and edge during surgery for GBM ( $\mathrm{n}=3$ patients). GBM core tissue was derived from areas within Gd-enhancing lesions, whereas 


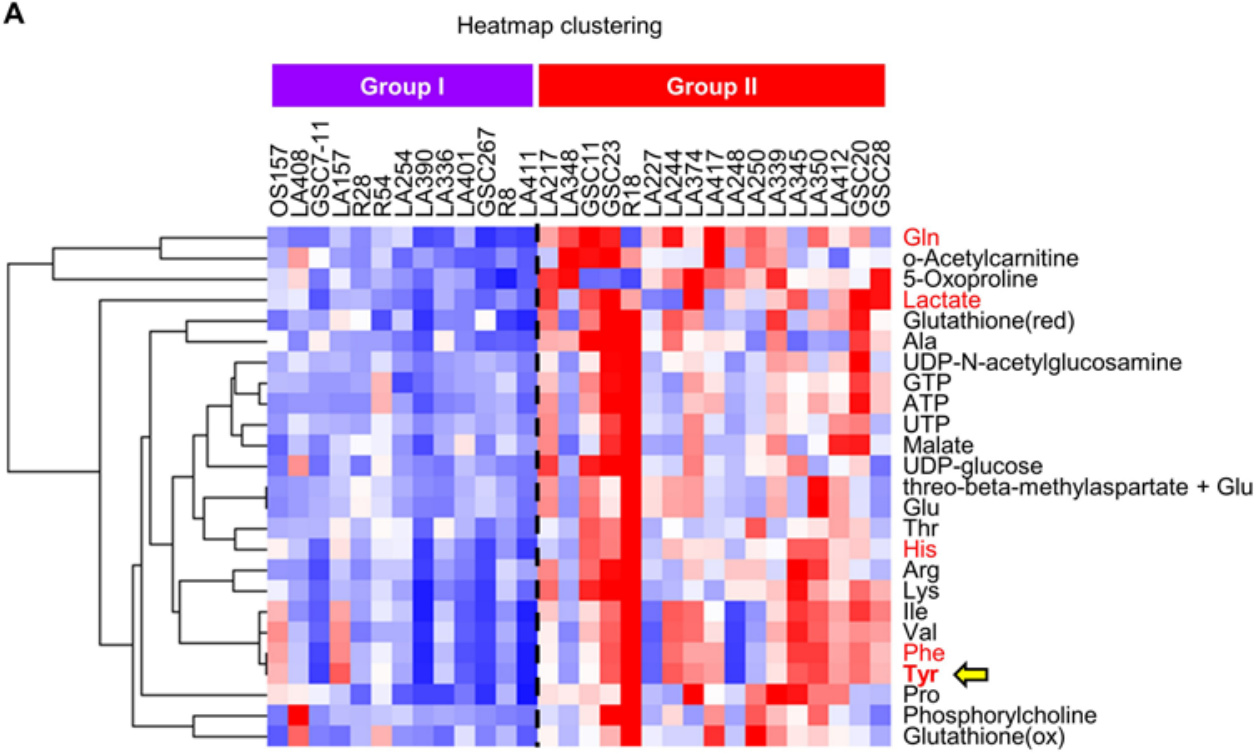

B

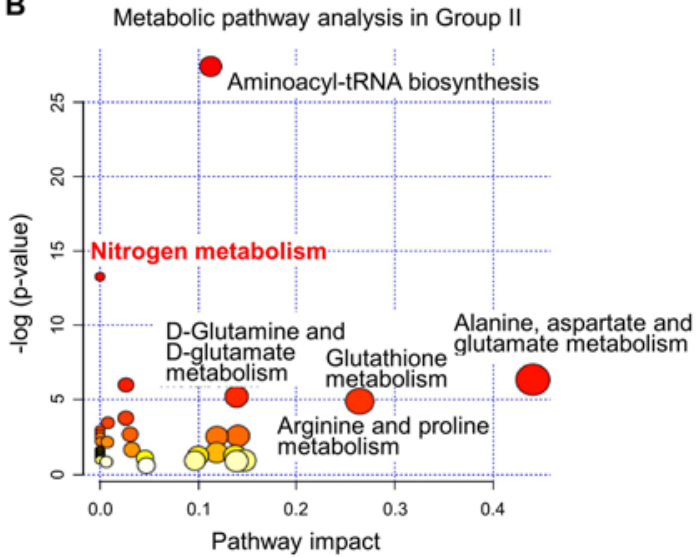

C

\begin{tabular}{lccc}
\multicolumn{4}{c}{ Upregulated pathway in Group II } \\
\hline \multicolumn{1}{c}{ Pathway name } & Total & Hits & p-value \\
\hline Aminoacyl-tRNA biosynthesis & 75 & 12 & $1.2842 \mathrm{E}-12$ \\
Nitrogen metabolism & 39 & 6 & $1.7182 \mathrm{E}-6$ \\
Glutathione metabolism & 38 & 4 & $5.2717 \mathrm{E}-4$ \\
Alanine, aspartate and glutamate metabolism & 24 & 3 & 0.0017 \\
Valine, leucine and isoleucine biosynthesis & 27 & 3 & 0.0025 \\
D-Glutamine and D-glutamate metabolism & 11 & 2 & 0.0054 \\
Arginine and proline metabolism & 77 & 4 & 0.0073 \\
Pyrimidine metabolism & 60 & 3 & 0.0229 \\
Phenylalanine, tyrosine and tryptophan biosynthesis & 27 & 2 & 0.0310 \\
\hline
\end{tabular}

FIG. 5. Metabolic analysis of GIC spheres. A: Heatmap of $30 \mathrm{GIC}$ spheres with an unsupervised hierarchical clustering of metabolites (column) per each GIC sphere (row). Red indicates higher expression, while blue indicates lower expression. B: Bubble plot for significance and enrichment of metabolic pathways in group II GIC spheres. C: Table detailing upregulated metabolic pathways in group II GIC spheres.

edge tissue was derived from areas outside of the enhancing portions of the tumors sampled (i.e., within T2-weighted change; Fig. 7A). Of note, GBM core and edge tissues exhibited distinct microscopic and macroscopic properties. The core consistently demonstrated pseudopalisading with areas of necrosis, whereas edge tissue demonstrated hypervascularity with a microscopic profile somewhat similar to that of normal brain. Interestingly, all 5 activated metabolites were highly expressed in tumor core tissue as compared to those in edge tissue (Fig. 7B). Similarly, 4-hydroxy-phenylpyruvate, a downstream metabolite of tyrosine, displayed higher expression within the tumor core as compared to that in the tumor edge (Fig. 7C). These data indicate that HGG tyrosine metabolism is preferentially activated within the tumor core as compared to that in the tumor edge (Fig. 7D). To further extend our analysis, we utilized the Ivy GAP database in an effort to identify spatially distinctive gene signatures across GBM regions (i.e., edge and core). ${ }^{33}$ Interestingly, tyrosine aminotrans- ferase (TAT) displayed higher expression within cellular tumor (CT) as opposed to the leading edge (LE), while the expression of phenylalanine hydroxylase (PAH) displayed the opposite trend, thereby suggesting a role for potential negative feedback (Fig. 7E). No other downstream enzymes (i.e., 4-hydroxyphenylpyruvate dioxygenase [HPD], homogentisate 1,2-dioxygenase [HGD]) displayed any significant differences despite similar trends in HPD. For further validation, we performed IHC for tyrosine-related proteins (PAH and TAT) within GBM tissues. As shown in Fig. 7F and G, we found that the tumor core has higher immunoreactivity for TAT than the tumor edge. These data raised the possibility that group III (tumor) and group II (GIC) may be related to metabolic activity in the tumor core. Finally, we sought the survival effects of TAT expression using web-based genomics analysis (https://hgserver1. amc.nl/cgi-bin/r2/main.cgi), and TAT showed a statistically significant clinical correlation with overall worse survival for both GBM and glioma patients (Fig. 7H). 
Yamashita et al.

A

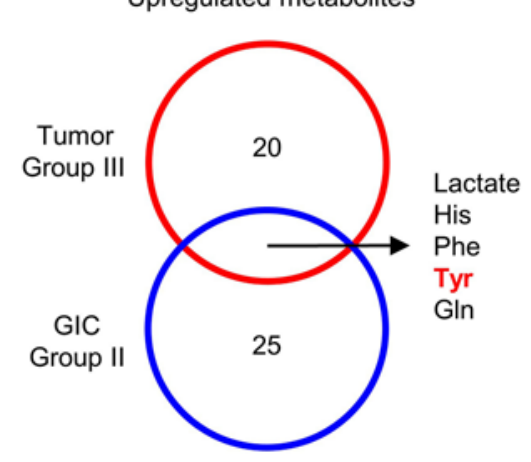

C

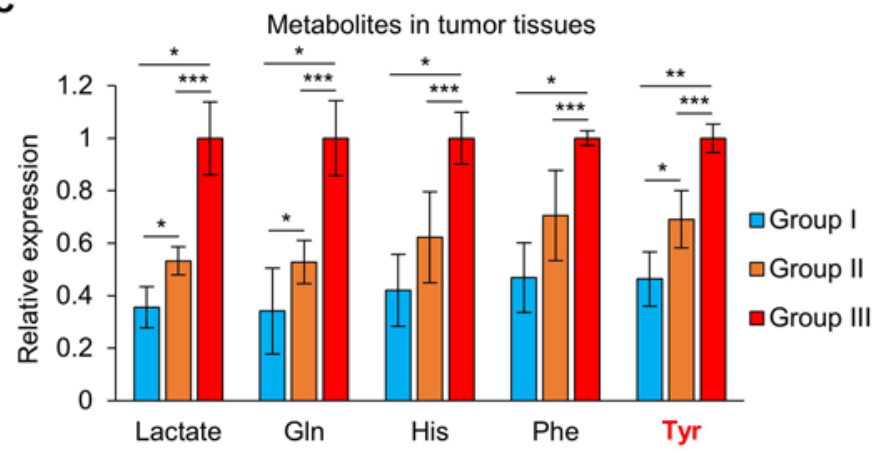

B

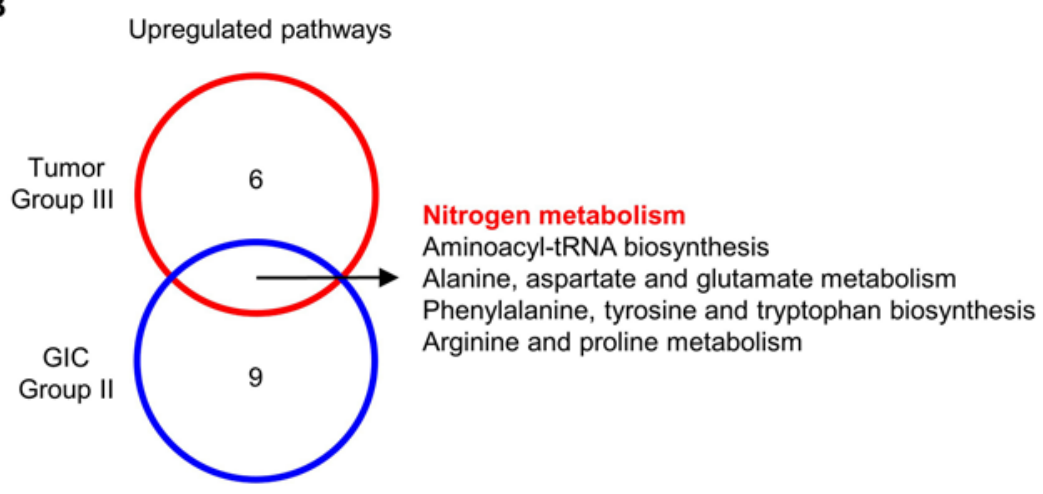

D
Metabolites in GIC spheres

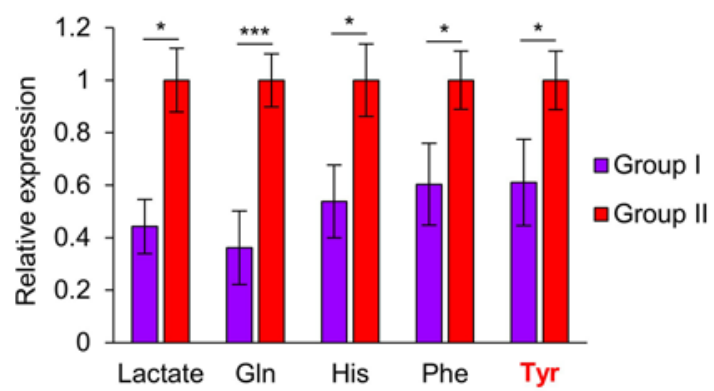

E

Nitrogen Metabolism

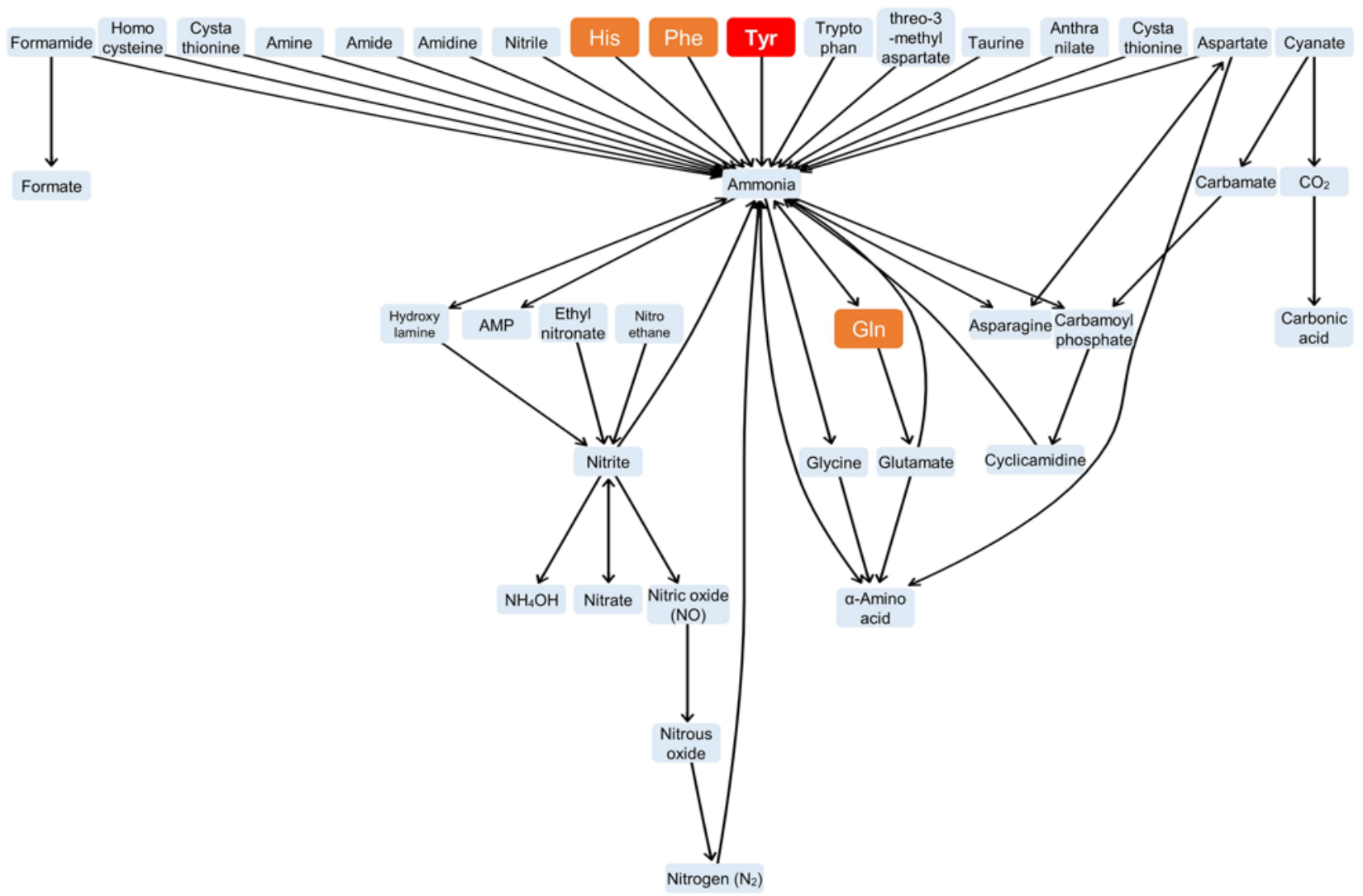

FIG. 6. Identification of upregulated metabolites and metabolic pathways. A: Venn diagram showing 5 upregulated metabolites in both group III tumor tissue samples and group II GIC spheres. B: Venn diagram showing 5 upregulated metabolic pathways in both group III tumor tissue samples and group II GIC spheres. C: Expression of 5 upregulated metabolites in 3 groups of tumor tissue samples. ${ }^{*} p<0.05,{ }^{* *} p<0.01$, and ${ }^{* * *} p<0.001$. D: Expression of 5 upregulated metabolites in 2 groups of GIC spheres. ${ }^{*} p<0.05$ and ${ }^{* * *} p<0.001$. E: Location of 4 upregulated metabolites in the nitrogen metabolism pathway. 
Yamashita et al.

A
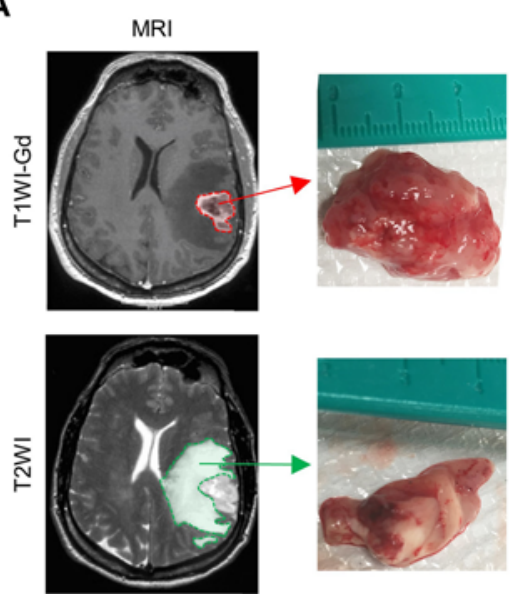

C

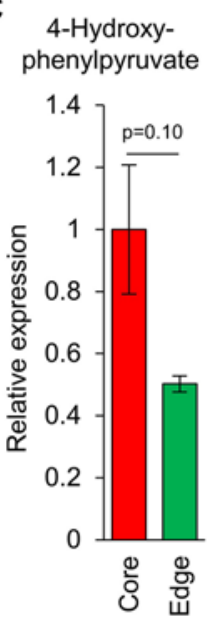

Core

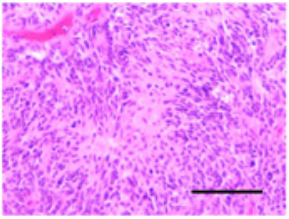

Edge

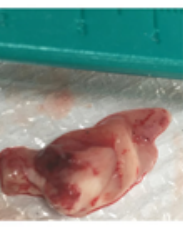

Tyrosine metabolism pathway

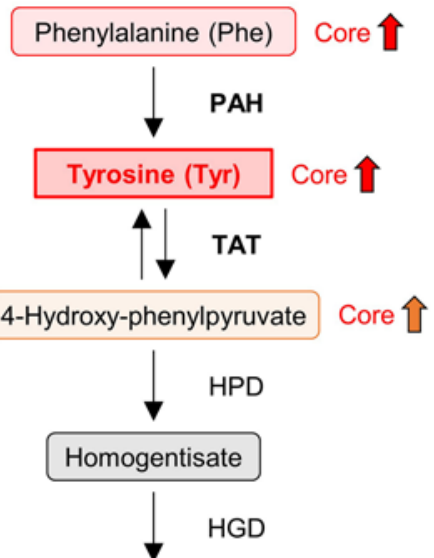

B

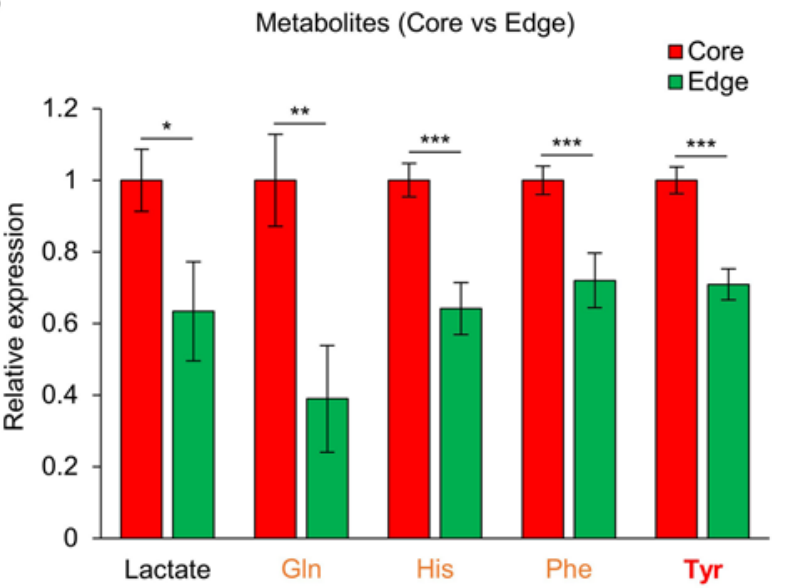

$\mathbf{F}$
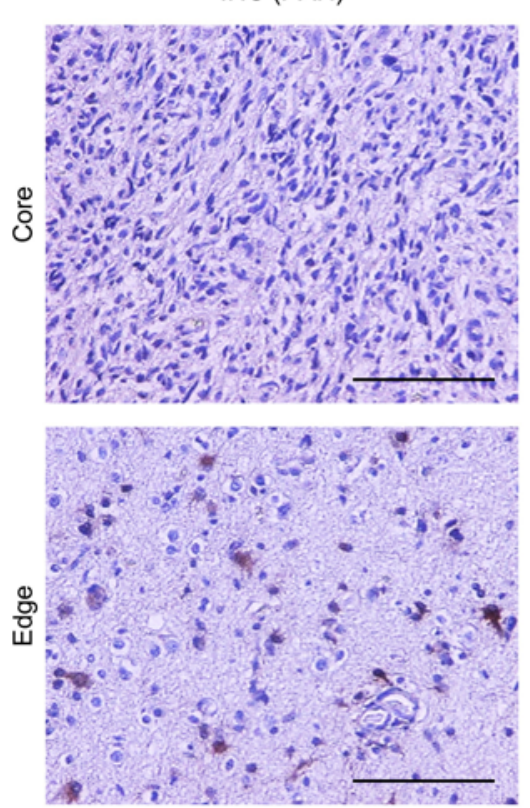

G
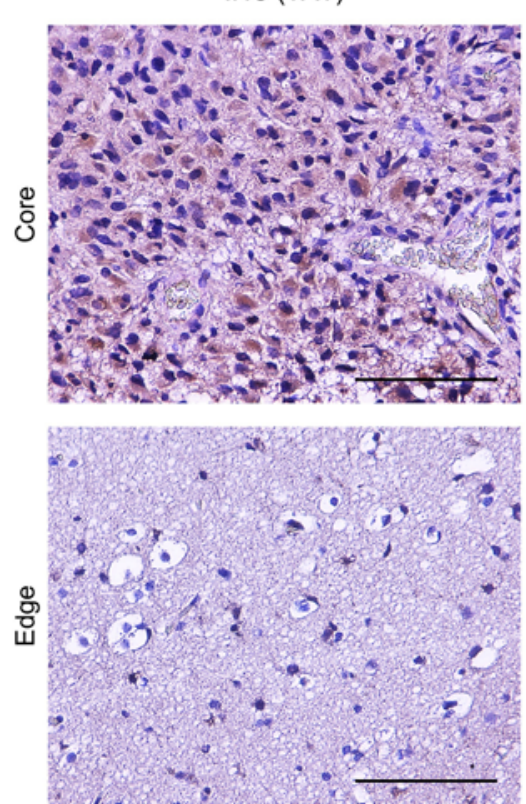

Ivy GAP

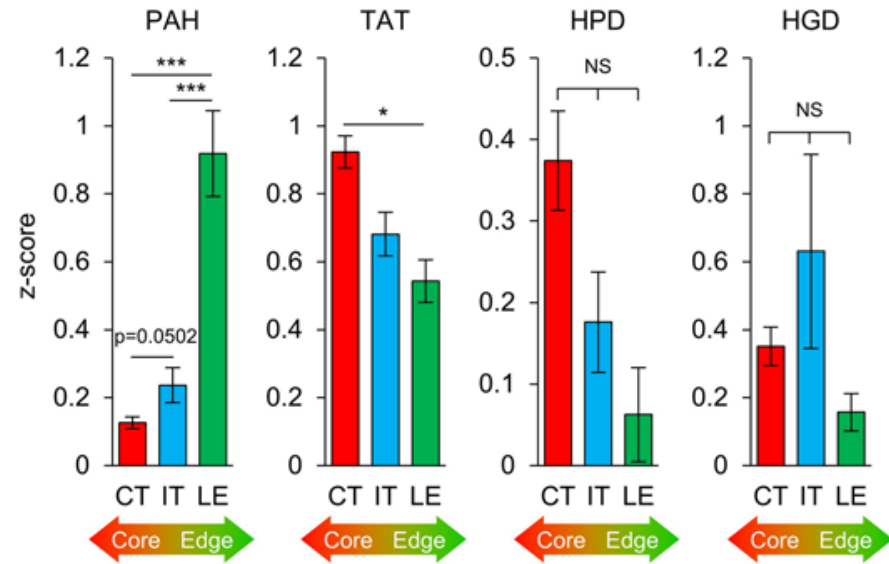

H

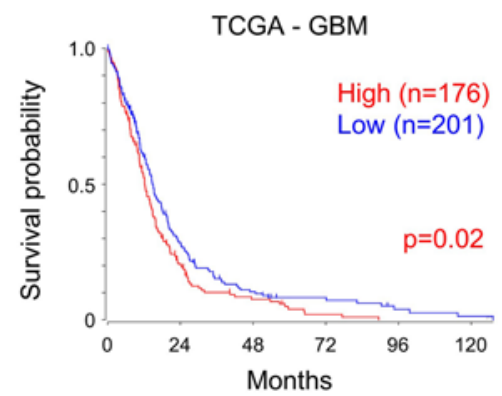

Glioma - French

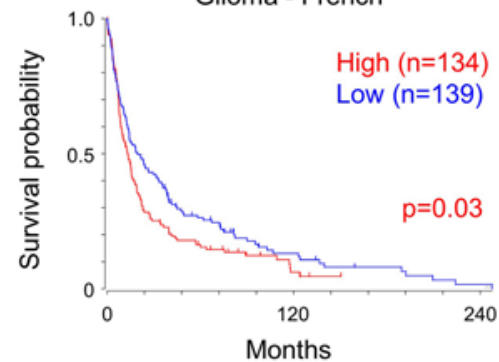

FIG. 7. Key molecules for abnormal tyrosine metabolism in HGG. A: Gd-enhancing T1-weighted (upper left) and T2-weighted (lower left) MR images obtained in a GBM patient, depicting the surgically resected core (upper center) and edge (lower center) of the GBM. H \& E staining of GBM core (upper right) and edge (lower right) tissues. Bar $=100 \mu \mathrm{m}$. B: Expression of 5 upregulated metabolites in core and edge GBM tissues. ${ }^{*} p<0.05,{ }^{* *} p<0.01$, and ${ }^{* *} p<0.001$. C: Expression of 4-hydroxy-phenylpyruvate in core and edge GBM tissues. FIG. 7. (continued) $\rightarrow$ 
FIG. 7. D: Key metabolites and enzymes in the tyrosine metabolism pathway. E: Data from RNA-seq from the Ivy GAP database showing the expression of 4 enzymes (PAH, TAT, HPD, and HGD). CT = cellular tumor; IT = infiltrating tumor; LE = leading edge; NS = not significant. ${ }^{*} p<0.05$ and ${ }^{* * *} p<0.001$. F: Representative IHC imaging for PAH within the GBM core and edge tissues. Bar $=100 \mu \mathrm{m}$. G: Representative IHC imaging for TAT within the GBM core and edge tissues. Bar $=100 \mu \mathrm{m}$. H: Kaplan-Meier survival curve comparing survival of GBM patients from the Glioblastoma-TCGA-395-MAS5.0u133a data set (upper) and Glioma-French-284-MAS5.0-u133p2 data set (lower), according to high or low TAT expression.

\section{Discussion}

In this study, we identified metabolic heterogeneity in both HGG tumor tissues and GIC sphere models. In so doing, we identified tyrosine metabolism as a possible therapeutic target in GBM given its differential upregulation within the tumor core. Furthermore, activation of TAT in tyrosine metabolism may in part be attributed to metabolic changes within the tumor core that manifest as therapyresistant and/or MES-like GBM. ${ }^{28}$

Currently, several novel metabolism-targeting therapies have been advanced as adjuvant treatments for gliomas..$^{35}$ Among them, metformin, an inhibitor of the mitochondrial respiratory chain (complex I) and activator of AMPactivated protein kinase (AMPK) ${ }^{41}$ has been employed in a number of randomized clinical trials (NCT02496741, ${ }^{29}$ NCT02149459, NCT02040376, NCT02780024, NCT 03243851, NCT03151772, and NCT01430351). Recently, a clinical trial was performed to focus on the effect of metformin in combination with chloroquine on $\mathrm{IDH}$-mutated solid tumors including gliomas. ${ }^{35}$ Moreover, disulfiram, an inhibitor of aldehyde dehydrogenase (ALDH), ${ }^{40}$ has been developed in several clinical trials (NCT02770378, ${ }^{14}$ NCT02678975, ${ }^{17}$ NCT03363659, NCT03151772, NCT 02715609, NCT01777919, ${ }^{18}$ NCT03034135, ${ }^{16}$ and NCT $\left.01907165^{18}\right) .{ }^{35}$ Other clinical trials are exploring the effects of ritonavir (NCT02770378), ${ }^{2,19}$ perifosine (NCT 00590954), and an energy-restricted ketogenic diet (metabolic nutritional therapy; NCT01535911) on GBM. ${ }^{34}$

The comprehensive metabolic analyses performed herein identified 5 elevated metabolites and 5 metabolic pathways. We ultimately focused on nitrogen and tyrosine metabolism since they represented the most significant pathways and metabolites. Tyrosine represents a critical part of nitrogen metabolism, and for decades nitrogen dysregulation cancer metabolism has been reported in multiple studies with various degrees of success. ${ }^{5,26}$ However, very few reports have centered on engaging tyrosine metabolism as a therapeutic target. ${ }^{24}$ Of note, 2-[2-nitro4-trifluoromethylbenzoyl]-1,3-cyclohexanedione (nitisinone [NTBC]), a potent inhibitor of 4-hydroxyphenylpyruvate dioxygenase in tyrosine metabolism, is widely used to treat tyrosinemia type $I^{7,15}$ Such therapies can ultimately be delivered locally by the resecting neurosurgeon, sparing off-target or systemically mediated effects. Future studies will be required to fully elucidate the role of tyrosine metabolism in the pathobiology of GBM.

Although numerous studies have reported that cancers spatially and temporally displayed a large degree of genetic and phenotypic heterogeneity, there are not many reports of metabolic heterogeneity in different spatial contexts. ${ }^{6,8,12}$ Moreover, only a few studies have investigated spatial metabolic heterogeneity in HGG..$^{37}$ Thus, our findings are novel and a challenge for targeting metabolites. However, it is prudent to note that the inhibition of a single metabolic pathway is likely overcome by cancer cells via the preexisting or therapy-reactive resistance mechanism..$^{9,21,39}$ Further assessment of the benefits related to the focal inhibition of tyrosine metabolism will be explored using regionally specified xenograft murine models.

Despite striking data showing that GBM has spatial metabolic heterogeneity, this study includes limitations. First, a small sample size weakened the statistical power of the analyses. Although we used 30 GIC models, 20 tumor tissue samples were obtained from only 5 HGG tissues, 4 GBM and 1 WHO grade III anaplastic oligodendroglioma. Second, we did not determine whether TAT is an actionable target within the tumor core of HGG. Therefore, a large-scale study of targeting metabolites would be necessary to confirm our findings.

\section{Conclusions}

This study demonstrated regional metabolic heterogeneity in HGG. Given that we identified significant metabolic inter- and intratumoral metabolic heterogeneity, our findings may help to guide new investigative and therapeutic approaches in GBM and other solid tumors.

\section{Acknowledgments}

We would like to thank all members of Dr. Nakano's laboratory (past and present) for their contributions that helped facilitate this work. In memory of A.O.C. (7/2019).

This study was supported by NIH grants to I.N. (R01NS083767, R01NS087913, R01CA183991, R01CA201402).

\section{References}

1. Agnihotri S, Zadeh G: Metabolic reprogramming in glioblastoma: the influence of cancer metabolism on epigenetics and unanswered questions. Neuro Oncol 18:160-172, 2016

2. Ahluwalia MS, Patton C, Stevens G, Tekautz T, Angelov L, Vogelbaum MA, et al: Phase II trial of ritonavir/lopinavir in patients with progressive or recurrent high-grade gliomas. J Neurooncol 102:317-321, 2011

3. Ahmed KA, Chinnaiyan P: Applying metabolomics to understand the aggressive phenotype and identify novel therapeutic targets in glioblastoma. Metabolites 4:740-750, 2014

4. Aisenberg AC, Reinafarje B, Potter VR: Studies on the Pasteur effect. I. General observations. J Biol Chem 224:10991113,1957

5. Argilés JM, Costelli P, Carbó N, Pallarés-Trujillo J, LópezSoriano FJ: Tumour growth and nitrogen metabolism in the host. Int J Oncol 14:479-486, 1999

6. Burrell RA, McGranahan N, Bartek J, Swanton C: The causes and consequences of genetic heterogeneity in cancer evolution. Nature 501:338-345, 2013

7. Chinsky JM, Singh R, Ficicioglu C, van Karnebeek CDM, Grompe M, Mitchell G, et al: Diagnosis and treatment of tyrosinemia type I: a US and Canadian consensus group review and recommendations. Genet Med 19:1380, 2017

8. Danhier P, Bański P, Payen VL, Grasso D, Ippolito L, Sonveaux $\mathrm{P}$, et al: Cancer metabolism in space and time: be- 
yond the Warburg effect. Biochim Biophys Acta Bioenerg 1858:556-572, 2017

9. DeBerardinis RJ, Chandel NS: Fundamentals of cancer metabolism. Sci Adv 2:e1600200, 2016

10. Frolkis A, Knox C, Lim E, Jewison T, Law V, Hau DD, et al: SMPDB: the Small Molecule Pathway Database. Nucleic Acids Res 38:D480-D487, 2010

11. Fuhrer T, Heer D, Begemann B, Zamboni N: High-throughput, accurate mass metabolome profiling of cellular extracts by flow injection-time-of-flight mass spectrometry. Anal Chem 83:7074-7080, 2011

12. Gerlinger M, Rowan AJ, Horswell S, Math M, Larkin J, Endesfelder D, et al: Intratumor heterogeneity and branched evolution revealed by multiregion sequencing. N Engl J Med 366:883-892, 2012

13. Griffin JL, Shockcor JP: Metabolic profiles of cancer cells. Nat Rev Cancer 4:551-561, 2004

14. Halatsch ME, Kast RE, Dwucet A, Hlavac M, Heiland T, Westhoff MA, et al: Bcl-2/Bcl-xL inhibition predominantly synergistically enhances the anti-neoplastic activity of a lowdose CUSP9 repurposed drug regime against glioblastoma. Br J Pharmacol 176:3681-3694, 2019

15. Holme E, Lindstedt S: Tyrosinaemia type I and NTBC (2-(2-nitro-4-trifluoromethylbenzoyl)-1,3-cyclohexanedione). J Inherit Metab Dis 21:507-517, 1998

16. Huang J, Chaudhary R, Cohen AL, Fink K, Goldlust S, Boockvar J, et al: A multicenter phase II study of temozolomide plus disulfiram and copper for recurrent temozolomide-resistant glioblastoma. J Neurooncol 142:537-544, 2019

17. Jakola AS, Werlenius K, Mudaisi M, Hylin S, Kinhult S, Bartek J Jr, et al: Disulfiram repurposing combined with nutritional copper supplement as add-on to chemotherapy in recurrent glioblastoma (DIRECT): study protocol for a randomized controlled trial. F1000 Res 7:1797, 2018

18. Karamanakos PN: Possible role for furazolidone in the treatment of glioblastoma multiforme. J BUON 18:1097, 2013

19. Kast RE, Karpel-Massler G, Halatsch ME: CUSP9* treatment protocol for recurrent glioblastoma: aprepitant, artesunate, auranofin, captopril, celecoxib, disulfiram, itraconazole, ritonavir, sertraline augmenting continuous low dose temozolomide. Oncotarget 5:8052-8082, 2014

20. Kim J, Lee IH, Cho HJ, Park CK, Jung YS, Kim Y, et al: Spatiotemporal evolution of the primary glioblastoma genome. Cancer Cell 28:318-328, 2015

21. Kinnaird A, Zhao S, Wellen KE, Michelakis ED: Metabolic control of epigenetics in cancer. Nat Rev Cancer 16:694707,2016

22. Klughammer J, Kiesel B, Roetzer T, Fortelny N, Nemc A, Nenning KH, et al: The DNA methylation landscape of glioblastoma disease progression shows extensive heterogeneity in time and space. Nat Med 24:1611-1624, 2018

23. Libby CJ, Tran AN, Scott SE, Griguer C, Hjelmeland AB: The pro-tumorigenic effects of metabolic alterations in glioblastoma including brain tumor initiating cells. Biochim Biophys Acta Rev Cancer 1869:175-188, 2018

24. Lim W, Song G: Characteristics, tissue-specific expression, and hormonal regulation of expression of tyrosine aminotransferase in the avian female reproductive tract. Domest Anim Endocrinol 57:10-20, 2016

25. Mao P, Joshi K, Li J, Kim SH, Li P, Santana-Santos L, et al: Mesenchymal glioma stem cells are maintained by activated glycolytic metabolism involving aldehyde dehydrogenase 1A3. Proc Natl Acad Sci U S A 110:8644-8649, 2013

26. Márquez J, Sánchez-Jiménez F, Medina MA, Quesada AR, Núñez de Castro I: Nitrogen metabolism in tumor bearing mice. Arch Biochem Biophys 268:667-675, 1989

27. Medes G, Friedmann B, Weinhouse S: Fatty acid metabolism. VIII. Acetate metabolism in vitro during hepatocar- cinogenesis by p-dimethylaminoazobenzene. Cancer Res 16:57-62, 1956

28. Minata M, Audia A, Shi J, Lu S, Bernstock J, Pavlyukov MS, et al: Phenotypic plasticity of invasive edge glioma stem-like cells in response to ionizing radiation. Cell Rep 26:18931905 e1897, 2019

29. Molenaar RJ, Coelen RJS, Khurshed M, Roos E, Caan MWA, van Linde ME, et al: Study protocol of a phase IB/II clinical trial of metformin and chloroquine in patients with IDH1-mutated or IDH2-mutated solid tumours. BMJ Open 7:e014961, 2017

30. Mooney J, Bernstock JD, Ilyas A, Ibrahim A, Yamashita D, Markert JM, et al: Current approaches and challenges in the molecular therapeutic targeting of glioblastoma. World Neurosurg 129:90-100, 2019

31. Nakano I: Stem cell signature in glioblastoma: therapeutic development for a moving target. J Neurosurg 122:324-330, 2015

32. Pavlova NN, Thompson CB: The emerging hallmarks of cancer metabolism. Cell Metab 23:27-47, 2016

33. Puchalski RB, Shah N, Miller J, Dalley R, Nomura SR, Yoon JG, et al: An anatomic transcriptional atlas of human glioblastoma. Science 360:660-663, 2018

34. Schwartz K, Chang HT, Nikolai M, Pernicone J, Rhee S, Olson K, et al: Treatment of glioma patients with ketogenic diets: report of two cases treated with an IRB-approved energyrestricted ketogenic diet protocol and review of the literature. Cancer Metab 3:3, 2015

35. Seliger C, Hau P: Drug repurposing of metabolic agents in malignant glioma. Int J Mol Sci 19:19, 2018

36. Spratlin JL, Serkova NJ, Eckhardt SG: Clinical applications of metabolomics in oncology: a review. Clin Cancer Res 15:431-440, 2009

37. Stadlbauer A, Zimmermann M, Doerfler A, Oberndorfer S, Buchfelder M, Coras R, et al: Intratumoral heterogeneity of oxygen metabolism and neovascularization uncovers 2 survival-relevant subgroups of IDH1 wild-type glioblastoma. Neuro Oncol 20:1536-1546, 2018

38. Stupp R, Mason WP, van den Bent MJ, Weller M, Fisher $\mathrm{B}$, Taphoorn MJ, et al: Radiotherapy plus concomitant and adjuvant temozolomide for glioblastoma. $\mathbf{N}$ Engl J Med 352:987-996, 2005

39. Sullivan LB, Gui DY, Vander Heiden MG: Altered metabolite levels in cancer: implications for tumour biology and cancer therapy. Nat Rev Cancer 16:680-693, 2016

40. Triscott J, Rose Pambid M, Dunn SE: Concise review: bullseye: targeting cancer stem cells to improve the treatment of gliomas by repurposing disulfiram. Stem Cells 33:10421046,2015

41. Vancura A, Bu P, Bhagwat M, Zeng J, Vancurova I: Metformin as an anticancer agent. Trends Pharmacol Sci 39:867878, 2018

42. Warburg O: On the origin of cancer cells. Science 123:309314,1956

43. Wishart DS, Tzur D, Knox C, Eisner R, Guo AC, Young N, et al: HMDB: the Human Metabolome Database. Nucleic Acids Res 35:D521-D526, 2007

44. Yan H, Parsons DW, Jin G, McLendon R, Rasheed BA, Yuan W, et al: IDH1 and IDH2 mutations in gliomas. N Engl J Med 360:765-773, 2009

45. Zhao S, Lin Y, Xu W, Jiang W, Zha Z, Wang P, et al: Gliomaderived mutations in IDH1 dominantly inhibit IDH1 catalytic activity and induce HIF-1 $\alpha$. Science 324:261-265, 2009

\section{Disclosures}

Dr. Bernstock has positions and equity in CITC Ltd. and Avidea Technologies and is a member of the Scientific Advisory Board for POCKiT Diagnostics. 


\section{Author Contributions}

Conception and design: Nakano. Acquisition of data: Yamashita, Sadahiro, Mohyeldin, Yamaguchi, Flanary, Hackney, Zamboni,

Kim. Analysis and interpretation of data: Yamashita, Bernstock, Zamboni, Kim. Drafting the article: Yamashita, Bernstock,

Elsayed, Sadahiro. Critically revising the article: Nakano.

Reviewed submitted version of manuscript: all authors.

Approved the final version of the manuscript on behalf of all authors: Nakano. Statistical analysis: Yamashita, Zamboni, Kim. Administrative/technical/material support: Bhat, Kornblum. Study supervision: Nakano.

\section{Correspondence}

Ichiro Nakano: University of Alabama at Birmingham, AL. inakano@uabmc.edu. 